\title{
Patient reported outcomes in patients undergoing arthroscopic partial meniscectomy for traumatic or degenerative meniscal tears: comparative prospective cohort study
}

\author{
Jonas Bloch Thorlund, ${ }^{1}$ Martin Englund, ${ }^{2,3}$ Robin Christensen, ${ }^{4}$ Nis Nissen, ${ }^{5}$ Kenneth Pihl, ${ }^{1}$ \\ Uffe Jørgensen, ${ }^{6}$ Jeppe Schjerning,' L Stefan Lohmander ${ }^{2}$
}

'Department of Sports Science

and Clinical Biomechanics,

University of Southern

Denmark, Odense, Denmark

2Lund University, Faculty of

Medicine, Department of

Clinical Sciences Lund,

Orthopedics, Clinical

Epidemiology Unit, Lund,

Sweden

${ }^{3}$ Clinical Epidemiology Research and Training Unit, Boston

University School of Medicine,

Boston, MA, USA

${ }^{4}$ Musculoskeletal Statistics Unit,

Parker Institute, Bispebjerg and

Frederiksberg Hospital,

Copenhagen, Denmark

${ }^{5}$ Department of Orthopedics,

Lillebaelt Hospital, Kolding,

Denmark

${ }^{6}$ Department of Orthopedics and Traumatology, Odense

University Hospital, Odense, Denmark

${ }^{7}$ Department of Orthopedics, Lillebaelt Hospital, Vejle,

Denmark

Correspondence to: J B Thorlund jthorlund@health.sdu.dk

Cite this as: BMJ 2017;356:j356

http://dx.doi.org/10.1136/bmj.j356

Accepted: 06 January 2017

\section{ABSTRACT}

OBJECTIVES

To compare patient reported outcomes from before surgery to 52 weeks after surgery between individuals undergoing arthroscopic partial meniscectomy for traumatic meniscal tears and those for degenerative meniscal tears.

DESIGN

Comparative prospective cohort study.

SETTING

Four public orthopaedic departments in the Region of Southern Denmark. Participants were recruited between 1 February 2013 and 31 January 2014, and at one of the original four hospitals from 1 February 2014 to 31 January 2015.

\section{PARTICIPANTS}

Individuals selected from Knee Arthroscopy Cohort Southern Denmark, aged 18-55, and undergoing arthroscopic partial meniscectomy for a traumatic or degenerative meniscal tear (defined by a combination of age and symptom onset).

\section{INTERVENTIONS}

Both participant groups underwent arthroscopic partial meniscectomy for a meniscal tear, with operating surgeons recording relevant information on knee pathology. Patient reported outcomes were recorded via online questionnaires.

\section{MAIN OUTCOME MEASURES}

Primary outcome was the average between-group difference in change on four of five subscales of the

\section{WHAT IS ALREADY KNOWN ON THIS TOPIC}

Arthroscopic partial meniscectomy is routine surgery for both patients with traumatic and degenerative meniscal tears

High quality evidence shows only marginal short term benefit of arthroscopic partial meniscectomy above placebo or non-surgical treatment for middle aged and older individuals with degenerative meniscal tears, but no trial evidence on patients with traumatic tears

Arthroscopic partial meniscectomy is presumed to improve patient reported outcomes to a greater extent in individuals with traumatic tears than in those with degenerative tears

\section{WHAT THIS STUDY ADDS}

No clinically meaningful difference in improvement in patient reported outcomes was seen between patients undergoing arthroscopic partial meniscectomy for traumatic tears and those with degenerative tears

Randomised trials are needed to compare the effect of arthroscopic partial meniscectomy with non-operative treatment or a sham surgery procedure on traumatic tears knee injury and osteoarthritis outcome score (KOOS). The four subscales covered pain, symptoms, sport and recreational function, and quality of life $\left(\mathrm{KOOS}_{4}\right)$. A 95\% confidence interval excluding differences greater than 10 KOOS points between groups was interpreted as absence of a clinically meaningful difference. Analyses adjusted for age, sex, and body mass index. RESULTS

397 eligible adults ( $42 \%$ women) with a traumatic or degenerative meniscal tear $(n=141$, mean age 38.7 years (standard deviation 10.9); $n=256,46.6$ years (6.4); respectively) were included in the main analysis. At 52 weeks after arthroscopic partial meniscectomy, 55 (14\%) patients were lost to follow-up. Statistically, participants with degenerative meniscal tears had a significantly larger improvement in $\mathrm{KOOS}_{4}$ scores than those with traumatic tears (adjusted between-group difference -5.1 (95\% confidence interval -8.9 to -1.3 ); $\mathrm{P}=0.008$ ). In the analysis including $\mathrm{KOOS}_{4}$ score at all time points, a significant time-by-group interaction was observed in both the unadjusted $(P=0.025)$ and adjusted analysis $(P=0.024)$, indicating better self-reported outcomes in participants with degenerative tears. However, the difference between groups was at no time point considered clinically meaningful.

\section{CONCLUSIONS}

These results question the current tenet that patients with traumatic meniscal tears experience greater improvements in patient reported outcomes after arthroscopic partial meniscectomy than patients with degenerative tears.

TRIAL REGISTRATION

ClinicalTrials.gov identifier NCT01871272.

\section{Introduction}

Knee arthroscopy for a meniscal tear is one of the most commonly performed orthopaedic procedures. Systematic reviews and meta-analyses of randomised trials have found arthroscopic knee surgery to provide no better effect than that of placebo surgery, nor any added benefit to exercise for middle aged and older patients with degenerative meniscal tears. ${ }^{12}$ Furthermore, no corresponding randomised trials were identified comparing non-surgical treatment with arthroscopic knee surgery for patients with meniscal tears of traumatic origin. ${ }^{2}$

Traumatic meniscal tears usually occur in an otherwise healthy meniscus in younger sports active individuals and can be attributed to a specific event such as a sports related trauma. ${ }^{3}$ By contrast, degenerative (non-traumatic) tears 
are typically observed in middle aged and older people ${ }^{4}$ and associated with incipient knee osteoarthritis. ${ }^{5-7}$ Such tears are associated with mucoid degeneration ${ }^{8}$ and meniscal calcification, ${ }^{9}$ and risk factors include age, ${ }^{4}$ high body mass index, ${ }^{410}$ knee malalignment, ${ }^{11}$ and occupational kneeling, ${ }^{12}$ although the cause is not entirely clear. ${ }^{3}$ Despite differences in symptom onset, meniscal tissue quality, and age distribution of patients with traumatic and degenerative tears, the same treatment-arthroscopic partial meniscectomy-has typically been offered for patients with both tear types.

In most observational studies, meniscal tear type (that is, traumatic or degenerative) has rarely been taken into account. ${ }^{1314}$ Reports from the early 1980 s suggested poorer results in individuals with degenerative changes undergoing arthroscopic meniscectomy. ${ }^{15-17}$ More recent studies investigating the difference in outcome between individuals with traumatic or degenerative tears have reported conflicting results. One study, including participants younger than 40 with isolated horizontal tears (a rare tear type in this population), reported similar outcomes in individuals with traumatic and non-traumatic tears of this type, two years after surgery. ${ }^{18}$ Another study observed similar outcomes at one year after surgery, but poorer outcomes in individuals with degenerative tears than in those with traumatic tears four years after meniscectomy or meniscal repair. ${ }^{19}$ However, both these studies were retrospective and included a limited number of participants. ${ }^{1819}$ Lastly, one larger study reported better outcomes in individuals with traumatic tears than in those with degenerative meniscal tears, four years after surgery. However, the outcomes were assessed at clinical visits or by telephone interview, and not by validated patient reported outcomes measures. ${ }^{20}$ Taken together, solid evidence from larger prospective studies using validated outcomes is lacking to confirm the current presumption that individuals with traumatic tears have larger improvements in patient reported outcomes after arthroscopic partial meniscectomy than those with degenerative tears.

Thus, we aimed to compare patient reported outcomes from before surgery to 52 weeks after surgery between individuals undergoing arthroscopic partial meniscectomy for traumatic tears and those with tears of degenerative origin. We hypothesised that individuals undergoing arthroscopic partial meniscectomy for traumatic tears would have larger improvements in patient reported pain, symptoms, function, and quality of life than those with degenerative tears.

\section{Methods}

We followed the strengthening the reporting of observational studies in epidemiology (STROBE) guideline to report this comparative prospective cohort study. ${ }^{21}$ The study has been registered at ClinicalTrials.gov (NCT01871272).

\section{Participants}

Participants from Knee Arthroscopy Cohort Southern Denmark (KACS) were included for this study. ${ }^{22}$ KACS is a prospective cohort following adults undergoing knee arthroscopy for meniscal tears. Participants were recruited at four different public hospitals in Denmark between 1 February 2013 and 31 January 2014, and at one hospital (one of the original four hospitals) from 1 February 2014 to 31 January 2015.

The KACS cohort inclusion criteria were individuals aged at least 18 years old, referred for knee arthroscopy on suspicion of a meniscus tear by an orthopaedic surgeon (that is, based on clinical examination, injury history, and magnetic resonance imaging if considered necessary), able to read and understand Danish, and who had an email address.

Exclusion criteria were no meniscal tear at surgery, previous or planned reconstruction surgery of the anterior or posterior cruciate ligament in either knee, fracture(s) to the lower extremities within the last six months before recruitment, or inability to reply to online questionnaires (see below) because of mental impairment.

For the present analysis, only participants aged 55 years or younger at baseline and undergoing meniscal resection (that is, not repair) at surgery were included. This age limit was set to minimise the proportion of participants with more advanced stages of osteoarthritis. Written informed consent was obtained from all participants, although the regional scientific ethics committee of Southern Denmark waived the need for ethical approval. ${ }^{22}$

\section{Patient reported outcomes and symptom descriptions}

We collected participant characteristics and information about symptoms using online questionnaires before surgery (median 7 days, interquartile range 3-10 days) and 12 weeks and 52 weeks after surgery.

Knee injury and osteoarthritis outcome score (KOOS)_The score consists of five subscales covering pain, symptoms, function during daily activities, sport and recreational function, and quality of life. Each subscale ranges from 0 to 100 points, with 0 representing extreme knee problems and 100 representing no knee problems. ${ }^{23}$ The score was developed with the involvement of patients and is intended for individuals with knee injuries that can result in post-traumatic osteoarthritis such as meniscus injury, anterior cruciate ligament injury, and chondral injury. ${ }^{23} \mathrm{KOOS}_{4}$ is the mean score of four of the five KOOS subscales (that is, excluding the daily activities subscale that is known to display ceiling effects in younger and more active populations $^{24}$ ). The KOOS questionnaire has been validated in individuals undergoing arthroscopic partial meniscectomy, ${ }^{232526}$ and $\mathrm{KOOS}_{4}$ has been used in trials assessing the effect of knee surgery. ${ }^{27-29}$

The present study's main outcome was the betweengroup difference in change from baseline to 52 weeks in the mean score on $\mathrm{KOOS}_{4}$. The study protocol and trial registration stated that change from baseline to 52 weeks on all five KOOS subscales was the main outcome. ${ }^{22}$ However, before analysis, we decided to use $\mathrm{KOOS}_{4}$ as the only main outcome, to simplify 
interpretation. To assist the clinical interpretation of our main outcome $\left(\mathrm{KOOS}_{4}\right)$, all five KOOS subscales were included as secondary outcomes.

Patient acceptable symptom state (PASS) and treatment failure-Additional secondary outcomes were PASS and treatment failure, 52 weeks after surgery. Satisfaction with current knee function (that is, PASS) was assessed with the question "When you think of your knee function, will you consider your current condition as satisfying? By knee function, you should take into account your activities of daily living, sport and recreational activities, your pain and other symptoms and your quality of life" (response options “yes” or "no"). This question has been used to assess PASS ${ }^{30}$ in individuals with knee injury. ${ }^{31}$ Participants not satisfied with current knee function at 52 weeks after surgery (who replied "no" to the PASS question) were then asked to complete a second question relating to treatment failure: "Would you consider your current state as being so unsatisfactory that you consider the treatment to have failed?" (response options "yes" or "no"). ${ }^{31}$

Symptom onset-Symptom onset was assessed with the question "How did the knee pain/problems for which you are now having surgery develop (choose the answer that best matches your situation)?" Response options were "The pain/problems have slowly evolved over time," "As a result of a specific incident (i.e. kneeling, sliding and/or twisting of the knee or the like, i.e. semi-traumatic onset)," or "As a result of a violent incident (i.e. during sports, a crash, collision or the like, i.e. traumatic onset)."

Symptom duration-Symptom duration was assessed with the question "How long have you had your knee pain/knee problems for which you are now having surgery?"

Mechanical symptoms-Presence and frequency of mechanical symptoms (that is, the sensation of catching or locking of the knee) was assessed with the question "How often have you experienced catching or locking of the knee, which is about to undergo surgery?" Five response options ranged from "never" to "daily".

\section{Structural pathology}

Information about meniscal tear type, tear placement (medial or lateral compartment), meniscal tissue quality (non-degenerative or degenerative), and cartilage defects was recorded by the operating surgeon at arthroscopy. A modified version of the International Society of Arthroscopy, Knee Surgery, and Orthopaedic Sports Medicine (ISAKOS) classification of meniscal tears questionnaire ${ }^{32}$ was used. Cartilage was also scored using the International Cartilage Repair Society (ICRS) grading system. ${ }^{33}$ ICRS cartilage scores range from 0 to 4 ( $0=$ normal cartilage; $4=$ very severe cartilage lesions). The inter-rater reliability for meniscal tear type and tissue quality has been reported to be good to moderate ( $\kappa$ coefficients of 0.72 and 0.47 , respectively) ${ }^{32}$ and good for ICRS cartilage grading (intraclass correlation 0.83). ${ }^{34}$ ICRS cartilage grade scoring from each knee joint compartment was added together to a 0-12 score for use as a covariate in the sensitivity analysis. Information registered by surgeons on the modified ISAKOS questionnaire was transferred from paper format to electronic format by automated forms processing, which has been validated as an alternative to double entry of data. ${ }^{35}$

\section{Categorisation of traumatic versus degenerative meniscal tears}

Traumatic meniscal tears were defined for those participants aged 18-34 and who replied that symptoms evolved as a result of a specific or violent incident, and for those aged 35-55 who replied that symptoms evolved as a result of a violent incident. Degenerative meniscal tears were defined for those participants aged 18-34 who replied that symptoms evolved slowly over time, and for those aged 35-55 who replied that symptoms evolved as a result of a specific incident or evolved slowly over time.

The definitions above were changed slightly before analysis from what was outlined in the study protocol, ${ }^{22}$ because some participants aged 18-55 were not categorised as having either a traumatic or degenerative tear by the previous definition. Furthermore, the criterion on duration of symptoms was relaxed because this was likely to be affected by referral time to the orthopaedic department.

\section{Statistics}

Descriptive statistics were presented as means with standard deviations, medians with interquartile ranges, and numbers with percentages as appropriate.

As reported in the study protocol, a participant flow with 200 participants in the degenerative group and 100 participants in the traumatic group would provide 0.99 power to detect an eight point difference in $\mathrm{KOOS}_{4}$, assuming a common standard deviation of 15 and a significance level of 0.05.22 The main outcome (betweengroup difference in $\mathrm{KOOS}_{4}$ change from baseline to 52 weeks) was analysed by a repeated measures mixed linear model (restricted maximum likelihood estimation (REML)) with patient nested within surgery site as random effects, and group (traumatic $v$ degenerative meniscal tears) and time (baseline, 12 weeks, and 52 weeks) as fixed effects. ${ }^{36}$ We changed to the current analysis (from the analysis of covariance approach presented in the protocol) because the mixed model approach (including all available data at all time points) using REML is considered a valid option to create unbiased parameter estimates and standard errors, and takes into account that repeated measures are non-independent. ${ }^{22}$

Adjusted models included age, sex, and body mass index as covariates because these were prespecified as being potential confounding factors. The same analysis approach was used for all secondary KOOS subscales analyses. For the main outcome $\left(\mathrm{KOOS}_{4}\right)$, we also tested the interaction between group and time to assess the difference in change over time including all time points. For all models, residual plots of fixed effects assessed the normal distribution of residuals and independence 
of predicted values. We used plots of best linear unbiased predictions to assess model assumptions of random effects. Results were reported as mean group scores and differences, with 95\% confidence intervals.

The minimal clinically important difference on KOOS was considered to be $8-10$ points. ${ }^{37}$ In the present study, a 95\% confidence interval excluding differences greater than 10 KOOS points between groups was interpreted as indicating the absence of a clinically meaningful difference between groups, similar to previous randomised trials using $\mathrm{KOOS}_{4}$ as the primary outcome..$^{27-29}$ We also conducted sensitivity analyses by using the degree of cartilage defects as a covariate in addition to age, sex, and body mass index for the main outcome, and included all participant characteristics with $\mathrm{P}<0.10$ and a fully adjusted model. Further sensitivity analyses assessed the robustness of the results with alternative definitions of traumatic and degenerative meniscal tears. Lastly, we used a non-responder imputation approach (that is, baseline observation carried forward) and a best or worst case scenario analysis as sensitivity analyses. The best or worse case scenario analysis involved imputing 25th

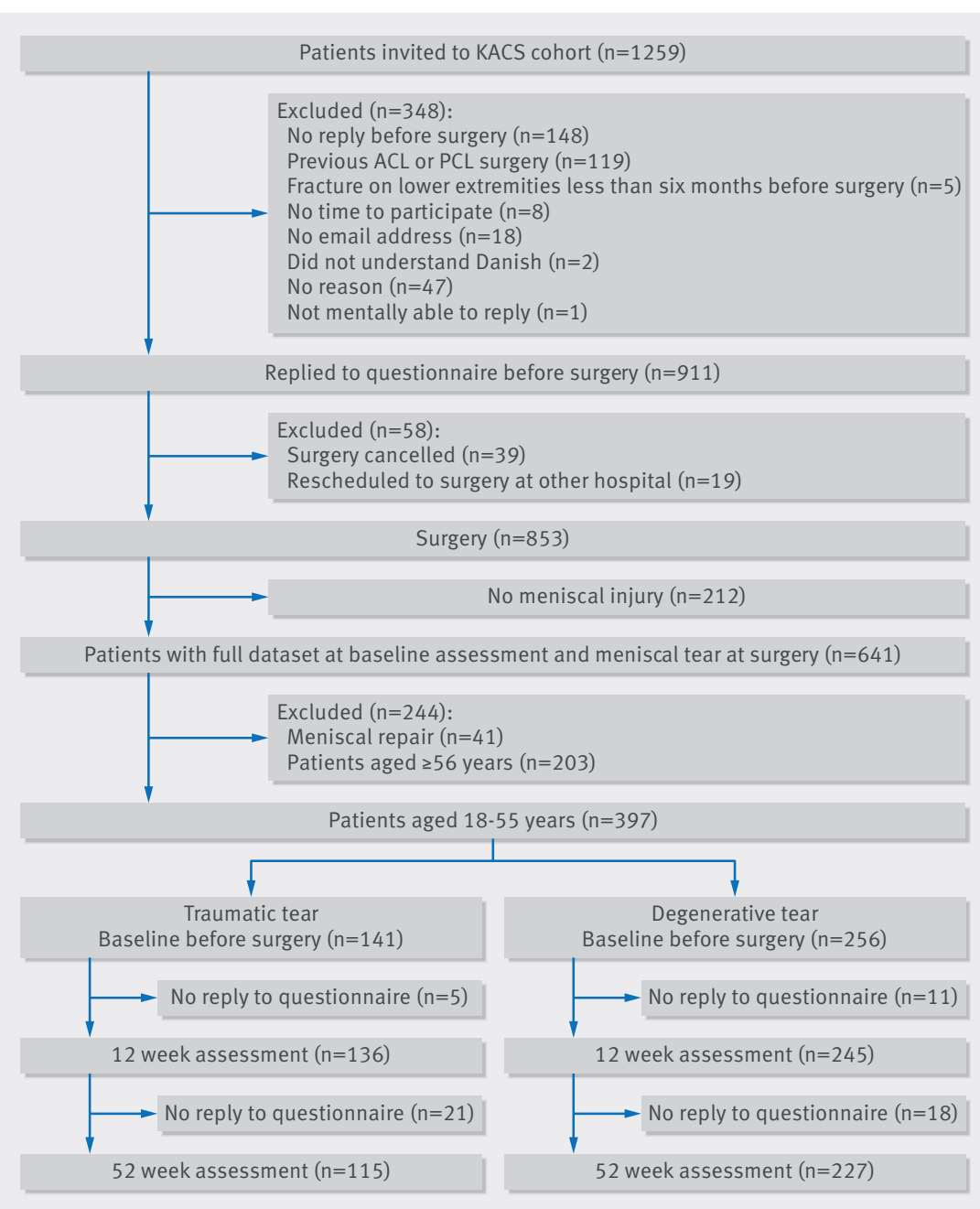

Fig 1 | Study flowchart, for participants with traumatic and degenerative meniscal tears. KACS=Knee Arthroscopy Cohort Southern Denmark; ACL=anterior cruciate ligament; $\mathrm{PCL}=$ posterior cruciate ligament percentile data from participants with available data at 12 week and 52 week follow-up for the degenerative tear group and 75th percentile data from the traumatic tear group (and vice versa)).

Differences in proportions of participants replying yes or no to the PASS question between participants with traumatic and degenerative meniscal tears were tested by the $\chi^{2}$ test and the calculation of risk differences with 95\% confidence intervals. We did similar analyses to test the difference in proportion of participants with traumatic and degenerative meniscal tears who indicated treatment failure ("yes" or "no" responses), from the participants who replied "no" to the PASS question. Stata 14.1 was used for all analysis.

\section{Patient involvement}

No patients were involved in setting the research question or the outcome measures, nor were they involved in developing plans for recruitment, design, or implementation of the study. No patients were asked to advise on interpretation or writing up of results. We plan to disseminate the results of the study in lay language in press and for patient interest groups.

\section{Results}

Of 641 participants who replied to the baseline questionnaire and had a meniscal tear at surgery, constituting the KACS baseline sample (fig 1), 244 were excluded for this analysis owing to meniscal repair $(n=41)$ or being 56 years or older $(n=203)$. The remaining 397 participants were defined as having a traumatic $(n=141)$ or degenerative meniscal tear $(n=256)$ according to the prespecified criteria. At the 52 week assessment, 55 (14\%) participants had been lost to follow-up. Participants lost to follow-up were similar to those retained in the study, although those with traumatic tears $(n=26)$ self-reported statistically significantly worse outcomes on most KOOS subscales at the baseline assessment before surgery (supplementary table 1).

Participants with degenerative tears were on average older, had a higher proportion of medial meniscal tears, and had more severe cartilage defects in the medial tibiofemoral compartment than those with traumatic tears. Similar levels of self-reported outcomes on all KOOS subscales were observed between groups at baseline before surgery (table 1).

In the main analysis, the degenerative tear group had a significantly greater improvement in $\mathrm{KOOS}_{4}$ score from before surgery to 52 weeks after surgery, compared with the traumatic tear group (crude mean difference -5.3 (95\% confidence interval -9.1 to -1.5 ); adjusted mean difference -5.1 ( -8.9 to -1.3$) ; \mathrm{P}=0.008$; table 2$)$. In the analysis including $\mathrm{KOOS}_{4}$ score at all time points, a significant time-by-group interaction was observed in both the unadjusted $(\mathrm{P}=0.025)$ and adjusted analysis $(\mathrm{P}=0.024)$, indicating better self-reported outcomes in participants with degenerative tears (fig 2). At no time point did the $95 \%$ confidence interval exceed the prespecified 10 point difference that was considered clinically relevant. Similar findings of no clinically relevant difference between groups were observed for all KOOS 
Table 1 | Baseline characteristics of participants with traumatic and degenerative meniscal tears

\begin{tabular}{|c|c|c|c|}
\hline & $\begin{array}{l}\text { Traumatic meniscal } \\
\text { tear }(n=141)\end{array}$ & $\begin{array}{l}\text { Degenerative meniscal } \\
\text { tear }(n=256)\end{array}$ & Difference $(95 \% \mathrm{Cl})$ or $\mathrm{P}$ \\
\hline Age (years; mean (standard deviation)) & $38.7(10.9)$ & $46.6(6.4)$ & $-7.9(-6.2$ to -9.6$)$ \\
\hline Female (No (\%)) & $53(38)$ & $113(44)$ & $-7(-17$ to 3$)$ \\
\hline Body mass index (mean (standard deviation)) & $27.1(4.9)$ & $27.6(4.6)$ & $-0.5(-1.4$ to 0.5$)$ \\
\hline \multicolumn{4}{|l|}{ Symptom onset (No (\%)): } \\
\hline Slowly evolved over time & 0 & $122(48)$ & \multirow{3}{*}{$<0.001$} \\
\hline Semi-traumatic & $20(14)$ & $134(52)$ & \\
\hline Traumatic & $121(86)$ & 0 & \\
\hline \multicolumn{4}{|l|}{ Mechanical symptoms (No (\%)): } \\
\hline Never & $65(46)$ & $114(45)$ & \multirow{5}{*}{0.813} \\
\hline Monthly & $24(17)$ & $45(18)$ & \\
\hline Weekly & $9(6)$ & $24(9)$ & \\
\hline Several times a week & $25(18)$ & $38(15)$ & \\
\hline Daily & $18(13)$ & $35(14)$ & \\
\hline \multicolumn{4}{|l|}{ Duration of symptoms (o (\%)): } \\
\hline $0-3$ months & $41(29)$ & $45(18)$ & \multirow{5}{*}{$<0.001$} \\
\hline 4-6 months & $16(11)$ & $78(30)$ & \\
\hline 7-12 months & $34(24)$ & $55(21)$ & \\
\hline 13-24 months & $21(15)$ & $40(16)$ & \\
\hline$>24$ months & $29(21)$ & $38(15)$ & \\
\hline \multicolumn{4}{|l|}{ Compartment (No (\%)): } \\
\hline Medial & $81(57)$ & $220(86)$ & \multirow{3}{*}{$<0.001$} \\
\hline Lateral & $48(34)$ & $25(10)$ & \\
\hline Both & $12(9)$ & $11(4)$ & \\
\hline \multicolumn{4}{|l|}{ Tear type (No (\%)): } \\
\hline Longitudinal-vertical & $36(26)$ & $37(14)$ & \multirow{8}{*}{0.050} \\
\hline Horizontal & $9(6)$ & $17(7)$ & \\
\hline Radial & $6(4)$ & $21(8)$ & \\
\hline Vertical flap & $32(23)$ & $64(25)$ & \\
\hline Horizontal flap & $11(8)$ & $13(5)$ & \\
\hline Complex & $29(21)$ & $78(30)$ & \\
\hline Root tear & 0 & 1 & \\
\hline More than one tear type & $18(13)$ & $25(10)$ & \\
\hline \multicolumn{4}{|l|}{ Meniscal tissue quality (No (\%))*: } \\
\hline Non-degenerative & $87(62)$ & 99 (39) & \multirow{3}{*}{$<0.001$} \\
\hline Degenerative & $45(32)$ & $151(59)$ & \\
\hline Undetermined & $9(6)$ & $5(2)$ & \\
\hline \multicolumn{4}{|c|}{ ICRS cartilage grade (No (\%))-medial compartmentt: } \\
\hline Grade 0 & $65(47)$ & $68(27)$ & \multirow{5}{*}{$<0.001$} \\
\hline Grade 1 & $36(26)$ & $61(25)$ & \\
\hline Grade 2 & $21(15)$ & $42(17)$ & \\
\hline Grade 3 & $13(9)$ & $58(23)$ & \\
\hline Grade 4 & $2(1)$ & $19(8)$ & \\
\hline \multicolumn{4}{|c|}{ ICRS cartilage grade (No (\%))-lateral compartmentt: } \\
\hline Grade 0 & $73(53)$ & $121(49)$ & \multirow{5}{*}{0.736} \\
\hline Grade 1 & $46(34)$ & $82(33)$ & \\
\hline Grade 2 & $12(9)$ & $27(11)$ & \\
\hline Grade 3 & $5(4)$ & $14(6)$ & \\
\hline Grade 4 & $1(1)$ & $4(2)$ & \\
\hline ICRS cartilage grade (No (\%))-patellofemoral & & & \\
\hline Grade 0 & $71(52)$ & $100(40)$ & \\
\hline Grade 1 & $38(28)$ & $64(26)$ & \\
\hline Grade 2 & $15(11)$ & $47(19)$ & 0.058 \\
\hline Grade 3 & $11(8)$ & $26(10)$ & \\
\hline Grade 4 & $2(1)$ & $11(4)$ & \\
\hline KOOS scores (mean (standard deviation)) & & & \\
\hline $\mathrm{KOOS}_{4}$ & $46.4(16.4)$ & $45.5(15.0)$ & $0.9(-2.3$ to 4.1$)$ \\
\hline Pain & $57.1(20.6)$ & $54.4(17.5)$ & $2.7(-1.2$ to 6.5$)$ \\
\hline Symptoms & $59.4(18.6)$ & $59.4(18.9)$ & $0.1(-3.8$ to 4.0$)$ \\
\hline Activities of daily living & $66.4(21.0)$ & $63.7(19.1)$ & $2.7(-1.4$ to 6.8$)$ \\
\hline Sport and recreational activities & $28.4(23.8)$ & $26.5(21.3)$ & $2.0(-2.6$ to 6.6$)$ \\
\hline Quality of life & $40.6(16.5)$ & $41.7(14.8)$ & $-1.1(-4.3$ to 2.1$)$ \\
\hline
\end{tabular}

ICRS=International Cartilage Repair Society grading system.

*Missing data on meniscal tissue quality, $n=1$.

tMissing data on cartilage damage, $n=12$. 


\begin{tabular}{|c|c|c|c|c|c|c|c|}
\hline & \multicolumn{3}{|l|}{12 week follow-up } & \multicolumn{3}{|l|}{52 week follow-up } & \multirow{2}{*}{$\begin{array}{l}\text { Difference }(95 \% \\
\mathrm{CI}) \text { from baseline } \\
\text { to } 52 \text { week } \\
\text { follow-up, TT } v \text { DT }\end{array}$} \\
\hline & $\mathrm{TT}(\mathrm{n}=136)$ & DT $(n=245)$ & $\begin{array}{l}\text { Difference } \\
(95 \% \mathrm{Cl})\end{array}$ & $\operatorname{TT}(n=115)$ & DT $(n=227)$ & $\begin{array}{l}\text { Difference } \\
(95 \% \mathrm{Cl})\end{array}$ & \\
\hline \multicolumn{8}{|l|}{ Unadjusted KOOS } \\
\hline $\mathrm{KOOS}_{4}$ & $57.4(54.2$ to 60.6$)$ & 58.7 (56.3 to 61.1) & $-1.3(-5.3$ to 2.7$)$ & $61.8(58.5$ to 65.2$)$ & 66.2 (63.8 to 68.7$)$ & $-4.4(-8.5$ to -0.2$)$ & $-5.3(-9.1$ to -1.5$)$ \\
\hline Pain & $70.4(67.1$ to 73.7$)$ & $71.2(68.8$ to 73.7$)$ & $-0.8(-4.9$ to 3.3$)$ & 71.5 (68.1 to 75.0$)$ & 77.3 (74.8 to 79.8$)$ & $-5.7(-10.0$ to -1.4$)$ & $-8.4(-12.4$ to -4.4$)$ \\
\hline Symptoms & $67.1(63.8$ to 70.4$)$ & $71.0(68.5$ to 73.4$)$ & $-3.8(-7.9$ to 0.3$)$ & 72.3 (68.8 to 75.7$)$ & 76.3 (73.8 to 78.8) & $-4.0(-8.3$ to 0.3$)$ & $-4.1(-8.3$ to 0.1$)$ \\
\hline Activities of daily living & $77.3(74.1$ to 80.5$)$ & 78.3 (75.9 to 80.7$)$ & $-1.0(-5.1$ to 3.0$)$ & 79.9 (76.5 to 83.2) & 83.3 (80.8 to 85.7) & $-3.4(-7.6$ to 0.7$)$ & $-6.1(-9.7$ to -2.5$)$ \\
\hline Sport and recreational activities & $43.8(39.3$ to 48.2$)$ & $41.6(38.3$ to 45.0$)$ & $2.1(-3.4$ to 7.7$)$ & $49.4(44.6$ to 54.1$)$ & 51.5 (48.1 to 54.9) & $-2.1(-7.9$ to 3.7$)$ & $-4.1(-9.7$ to 1.5$)$ \\
\hline Quality of life & $48.3(45.0$ to 51.7$)$ & $51.0(48.5$ to 53.5$)$ & $-2.6(-6.8$ to 1.5$)$ & $54.5(51.0$ to 58.1$)$ & $59.9(57.3$ to 62.4$)$ & $-5.4(-9.7$ to 1.0$)$ & $-4.3(-8.7$ to 0.1$)$ \\
\hline \multicolumn{8}{|l|}{ Adjusted KOOS* } \\
\hline $\mathrm{KOOS}_{4}$ & $57.4(54.3$ to 60.5$)$ & $58.7(56.4$ to 61.0$)$ & $-1.3(-5.1$ to 2.6$)$ & $61.9(58.7$ to 65.2$)$ & $66.2(63.8$ to 68.5$)$ & $-4.2(-8.3$ to -0.2$)$ & $-5.1(-8.9$ to -1.3$)$ \\
\hline Pain & $70.4(67.2$ to 73.6$)$ & $71.2(68.8$ to 73.5$)$ & $-0.8(-4.7$ to 3.2$)$ & 71.7 (68.3 to 75.0$)$ & $77.2(74.8$ to 79.6$)$ & $-5.5(-9.7$ to -1.4$)$ & $-8.2(-12.2$ to -4.2$)$ \\
\hline Symptoms & 67.1 (63.8 to 70.3$)$ & 70.9 (68.5 to 73.3$)$ & $-3.9(-7.9$ to 0.2$)$ & 72.3 (68.9 to 75.7$)$ & 76.2 (73.8 to 78.7$)$ & $-3.9(-8.2$ to 0.3$)$ & $-4.0(-8.2$ to 0.2$)$ \\
\hline Activities of daily living & 77.3 (74.2 to 80.3) & 78.3 (76.0 to 80.5$)$ & $-1.0(-4.8$ to 2.8$)$ & 80.0 (76.8 to 83.1) & $83.2(80.9$ to 85.5$)$ & $-3.2(-7.1$ to 0.7$)$ & $-5.9(-9.5$ to -2.3$)$ \\
\hline Sport and recreational activities & 43.7 (39.4 to 48.0) & $41.6(38.4$ to 44.8$)$ & $2.1(-3.2$ to 7.5$)$ & $49.4(44.9$ to 54.0$)$ & $51.4(48.1$ to 54.7$)$ & $-1.9(-7.6$ to 3.7$)$ & $-3.8(-9.5$ to 1.7$)$ \\
\hline Quality of life & $48.3(45.0$ to 51.6$)$ & $51.0(48.5$ to 53.4$)$ & $-2.6(-6.7$ to 1.5$)$ & $54.6(51.1$ to 58.1$)$ & $59.9(57.3$ to 62.4$)$ & $-5.3(-9.6$ to -0.9$)$ & $-4.2(-8.5$ to 0.2$)$ \\
\hline
\end{tabular}

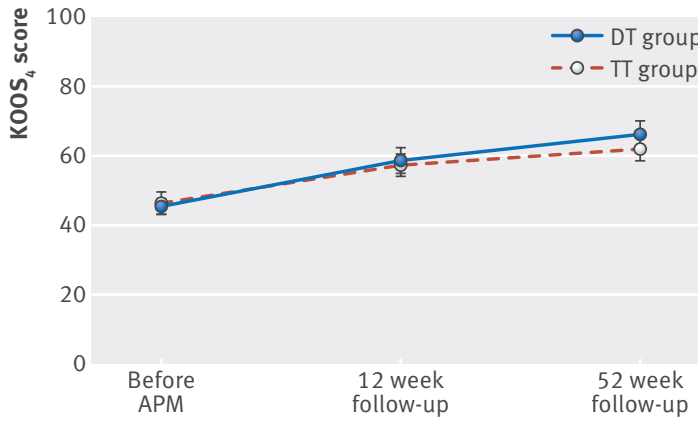

Fig 2 Mean score of four KOOS subscales (pain, symptoms, sport and recreational function, and quality of life $\left(\mathrm{KOOS}_{4}\right)$ ) assessed before arthroscopic partial meniscectomy (APM), and at 12 week and 52 week follow-up, for study participants with traumatic (TT) and degenerative (DT) meniscal tears. Data from model adjusted for age, sex, and body mass index. Bars indicate $95 \%$ confidence intervals. Group-by-time interaction from crude $(\mathrm{P}=0.025)$ and adjusted analysis $(\mathrm{P}=0.024)$

subscales apart from the pain subscale, which crossed the $95 \%$ confidence interval in favour of a larger clinically meaningful improvement in the degenerative tear group (table 2).

In the sensitivity analysis of the main outcome $\left(\mathrm{KOOS}_{4}\right)$, adding the degree of cartilage defects as a covariate did not change the interpretation of results, which was similar for analysis including all participant characteristics with $\mathrm{P}<0.10$ and the fully adjusted analysis (supplementary table 2).

Further sensitivity analyses testing different definitions of traumatic and degenerative meniscal tears did not change the interpretation of results. Analyses included different allocation of patients with semi-traumatic tears (supplementary tables 3 and 4), basing the tear definition on surgeon assessed meniscal tissue quality alone or in combination with symptom onset (supplementary tables 5 and 6, respectively), or using the original definition stated in the protocol (supplementary table 7).

We also compared the traumatic and degenerative tear groups with participants older than 55 (who were excluded from the present study; supplementary table 8). Again, no clinically relevant differences were observed between either tear group and the older participant group (supplementary fig 1). Lastly, sensitivity analysis using null responder imputation and assuming a best or worst case scenario of participants lost to follow-up did not alter the interpretation of data. However, the best case analysis in the degenerative tear group and worst case analysis in the traumatic tear group indicated the possibility of a clinically relevant larger improvement in the degenerative tear group (supplementary table 9).

A larger proportion of participants with degenerative tears were satisfied with their current knee function at 52 week follow-up than those with traumatic tears $(63 \%$ $v 52 \%$; table 3). However, a similar proportion in both groups were not satisfied with their knee function at 52 weeks and considered the treatment to have failed (35\% $v$ 41\%; table 3).

\section{Discussion}

Contrary to current opinion that individuals undergoing arthroscopic partial meniscectomy for traumatic meniscal tears have greater improvements in patient reported outcomes than those with degenerative meniscal tears, we found a significantly larger improvement in $\mathrm{KOOS}_{4}$ scores-indicating better patient reported outcomes-for participants with degenerative tears. However, the difference was small and did not reach the prespecified level of a clinically meaningful difference at any time point up to one year, except for the KOOS pain subscale. A larger proportion of participants with traumatic tears were not satisfied with current knee function at 52 week follow-up, compared with participants with degenerative tears. About $15 \%$ of 


\begin{tabular}{|c|c|c|c|}
\hline & $\begin{array}{l}\text { Traumatic meniscal } \\
\text { tear }(n=115)\end{array}$ & $\begin{array}{l}\text { Degenerative meniscal } \\
\text { tear }(n=227)\end{array}$ & $\begin{array}{l}\text { Risk difference } \\
(95 \% \mathrm{CI})\end{array}$ \\
\hline Satisfied with current knee function (PASS), yes/no response & $60(52) / 55(48)$ & $144(63) / 83(37)$ & $0.11(0.01$ to 0.22$)$ \\
\hline Treatment failure, yes/no response* & $19(35) / 36(65)$ & $34(41) / 49(59)$ & $-0.06(-0.23$ to 0.10$)$ \\
\hline
\end{tabular}

participants (53/342) were dissatisfied enough to consider that the treatment had failed, with no difference between groups.

\section{Strength and weaknesses of the study}

No randomised trials have compared the effects of arthroscopic partial meniscectomy with sham surgery or non-surgical treatment options such as exercise for traumatic meniscal tears. ${ }^{2}$ Knowledge on the natural time course of patient reported outcomes after arthroscopic partial meniscectomy is sparse in these patients. Most previous studies failed to account for symptom onset (that is, traumatic or non-traumatic), patient age, or concomitant injury to the anterior cruciate ligament. ${ }^{1314}$ Early reports suggested better results in the absence of degenerative changes when undergoing arthroscopic partial meniscectomy. ${ }^{15-17}$ More recent studies have shown conflicting results, but these have been limited by poor study quality or small sample size. ${ }^{18-2038}$ In the present study, participants were prospectively followed according to a prespecified protocol ${ }^{22}$ using a validated patient reported outcome measure, enabling us to compare the natural time course of patient reported outcomes in participants with traumatic and degenerative meniscal tears.

No consensus exists on the definitions of traumatic and degenerative tears, and there is a grey zone between the two. Therefore, we conducted several sensitivity analyses testing the robustness of the results by adjusting for meniscal and other structural knee joint pathologies observed at surgery, and by applying different definitions of traumatic and degenerative meniscal tears. Even though the level of statistical significance and the direction of the results varied slightly in these analyses, there was no clinically meaningful difference between groups (supplementary material).

The present study was powered to detect an eight point difference in improvement in KOOS scores between groups, because 8-10 KOOS points was considered a clinically relevant difference when the study was planned. ${ }^{37}$ However, there is no consensus on the specific value that constitutes a clinically relevant difference or change on the KOOS score. Before analysis, we decided to interpret a 95\% confidence interval excluding differences greater than 10 KOOS points between groups as absence of a clinically meaningful difference, as has been used in randomised trials comparing surgery with exercise therapy for patients with different knee pathologies..$^{27-29}$
We excluded participants aged 56 years or older because these individuals are likely to have more advanced stages of knee osteoarthritis. Excluding these participants could have led to better average KOOS scores in the degenerative tear group, because older age is associated with worse outcome after knee injury. ${ }^{39}$ However, sensitivity analysis showed that the $\mathrm{KOOS}_{4}$ time course did not differ between the degenerative tear group, participants aged 56 years or older, or the traumatic tear group (group-by-time interaction, $\mathrm{P}=0.080$ ).

Some participants were lost to follow-up. At 52 weeks, loss to follow-up was $18 \%$ and $11 \%$ for participants with traumatic and degenerative tears, respectively. In the traumatic tear group, participants lost to follow-up self-reported markedly poorer on four of five KOOS subscales at the baseline assessment before surgery, compared with those who remained in the study. The direction of the resulting bias due to loss to follow-up of these participants is uncertain. However, sensitivity analyses with null responder imputation or assuming the best or worst case scenario for patients lost to follow-up did not change the overall interpretation of data.

Participant age and sex distribution in the KACS cohort is similar to what has been reported for patients undergoing meniscal surgery in Denmark..$^{40}$ Nevertheless, participants having meniscal repair at surgery were excluded because we intended to compare patient reported outcomes of two distinct patient groups receiving the same type of treatment. Thus, the present results only apply to individuals having arthroscopic partial meniscectomy.

\section{Meaning of the study}

Participants self-reported substantial impairments on the KOOS questionnaire before arthroscopic partial meniscectomy. The levels of self-reported impairments before surgery were similar to previous reports on individuals with meniscal tears..$^{41}$ On average, participants reported improved patient reported outcomes (effect size >1.0) from baseline to 52 week follow-up. However, KOOS scores were still substantially lower at 52 weeks after surgery than population based data from Sweden on individuals aged 18-54. In particular, participants in the present study scored more than 25 KOOS points lower in the subscales of sport and recreational activities and quality of life than population based data. ${ }^{42}$

Studies on the effect of arthroscopic partial meniscectomy for individuals with degenerative meniscal tears have shown similar improvements as in individuals 
receiving sham surgery, ${ }^{43}$ independent of the presence or absence of self-reported mechanical symptoms. ${ }^{44}$ Exercise was recently shown to be as effective as arthroscopic partial meniscectomy to improve patient reported outcomes. ${ }^{29}$ Systematic reviews and meta-analyses have similarly reported no added benefit of arthroscopic partial meniscectomy or debridement in addition to exercise for individuals with degenerative meniscal tears. ${ }^{12}$

Acknowledging limitations provided by the observational nature of our study, it is noteworthy that the common presumption of better patient reported outcomes after surgery for younger individuals with traumatic tears compared with middle aged individuals with degenerative meniscal tears was not confirmed. Furthermore, almost half of participants with traumatic tears were not satisfied with their current knee function one year after arthroscopic partial meniscectomy.

It is unknown whether individuals with traumatic meniscal tears would have similar improvements in self-reported outcomes with exercise therapy as reported for individuals with degenerative meniscal tears. ${ }^{29}$ However, in a randomised trial on young, active individuals with acute injury to the anterior cruciate ligament, of which many had concomitant meniscal injuries, researchers observed a reduced need for reconstruction of the anterior cruciate ligament in those who received exercise as first line treatment. ${ }^{28}$ Avoiding arthroscopic partial meniscectomy could be important in relation to the risk of later development of knee osteoarthritis; a recent observational study reported the procedure to be associated with a greater risk of cartilage loss and incident knee osteoarthritis. ${ }^{45}$ Furthermore, patients who have had previous knee surgery undergo total knee replacement at a substantially younger age than those without previous knee surgery. ${ }^{46}$

\section{Unanswered questions and future research}

The common presumption that individuals with traumatic tears experience greater improvements in patient reported outcomes than those with degenerative tears after arthroscopic partial meniscectomy was not supported by our results. Given the lack of effect of arthroscopic partial meniscectomy compared with placebo surgery for degenerative meniscal tears, ${ }^{43}$ and the positive effects of exercise for patients with degenerative meniscal tears ${ }^{29}$ and anterior cruciate ligament injury, ${ }^{28}$ the efficacy of arthroscopic partial meniscectomy for traumatic meniscal tears should be compared in controlled trials with placebo or non-operative treatment such as exercise.

We thank all participating patients and orthopaedic surgeons, nurses, and secretaries at the Department of Orthopedics and Traumatology at Odense University Hospital (Odense and Svendborg) and Department of Orthopedics at Lillebaelt Hospital (Kolding and Vejle) for their assistance with patient recruitment and data collection.

Contributors: JBT, RC, LSL, and ME conceived and designed the study. $N N, U J$, and JS participated in the setup of the study, patient recruitment, and data collection. JBT and KP conducted the analysis. JBT drafted the first version of the manuscript. All authors helped in revising the manuscript and gave their final approval of the submitted version. JBT is the guarantor. All authors had full access to the data and take responsibility for the integrity of the data and the accuracy of the data analysis.

Funding: This study was supported by an individual postdoctoral grant (JBT) from the Danish Council for Independent Research/Medical Sciences and funds from the Region of Southern Denmark. The funders had no role in any part of the study or in any decision about publication.

Competing interests: All authors have completed the ICMJE uniform disclosure form at www.icmje.org/coi disclosure.pdf and declare: support from the Danish Council for Independent Research/Medical Sciences and the Region of Southern Denmark for the submitted work; the Parker Institute, Bispebjerg and Frederiksberg Hospital (RC) is supported by a core grant from the Oak Foundation (OCAY-13-309); no financial relationships with any organisations that might have an interest in the submitted work in the previous three years; no other relationships or activities that could appear to have influenced the submitted work.

Ethical approval: Written informed consent was obtained from all participants, although the regional scientific ethics committee of southern Denmark waived the need for ethical approval.

Data sharing: Full dataset to replicate the main analysis is available from the corresponding author on reasonable request.

The lead author affirms that this manuscript is an honest, accurate, and transparent account of the study being reported; that no important aspects of the study have been omitted; and that any discrepancies from the study as planned have been explained.

This is an Open Access article distributed in accordance with the Creative Commons Attribution Non Commercial (CC BY-NC 4.0) license, which permits others to distribute, remix, adapt, build upon this work non-commercially, and license their derivative works on different terms, provided the original work is properly cited and the use is non-commercial. See: http://creativecommons.org/licenses/ by-nc/4.0/.

1 Khan M, Evaniew N, Bedi A, Ayeni OR, Bhandari M. Arthroscopic surgery for degenerative tears of the meniscus: a systematic review and meta-analysis. CMA/ 2014;186:1057-64. doi:10.1503/ cmaj.140433

2 Thorlund JB, Juhl CB, Roos EM, Lohmander LS. Arthroscopic surgery for degenerative knee: systematic review and meta-analysis of benefits and harms. BMJ 2015;350:h2747. doi:10.1136/bmj.h2747.

3 Poehling GG, Ruch DS, Chabon SJ. The landscape of meniscal injuries. Clin Sports Med 1990;9:539-49.

4 Englund M, Guermazi A, Gale D, et al. Incidental meniscal findings on knee MRI in middle-aged and elderly persons. N Engl J Med 2008;359:1108-15. doi:10.1056/NEJMoa0800777.

5 Englund $M$. The role of the meniscus in osteoarthritis genesis. Rheum Dis Clin North Am 2008;34:573-9. doi:10.1016/j.rdc.2008.05.009.

6 Lohmander LS, Englund PM, Dahl LL, Roos EM. The long-term consequence of anterior cruciate ligament and meniscus injuries: osteoarthritis. Am J Sports Med 2007;35:1756-69. doi:10.1177/0363546507307396.

7 Englund M, Guermazi A, Roemer FW, et al. Meniscal tear in knees without surgery and the development of radiographic osteoarthritis among middle-aged and elderly persons: the Multicenter Osteoarthritis Study. Arthritis Rheum 2009;60:831-9. doi:10.1002 art.24383.

8 Stoller DW, Martin C, Crues JV 3rd, , Kaplan L, MinkJH. Meniscal tears: pathologic correlation with MR imaging. Radiology 1987;163:731-5. doi:10.1148/radiology.163.3.3575724.

9 Sun Y, Mauerhan DR. Meniscal calcification, pathogenesis and implications. Curr Opin Rheumatol 2012;24:152-7. doi:10.1097/ BOR.0b013e32834e90c1.

10 Baker P, Coggon D, Reading I, Barrett D, McLaren M, Cooper C. Sports injury, occupational physical activity, joint laxity, and meniscal damage. J Rheumatol 2002;29:557-63.

11 Englund M, Felson DT, Guermazi A et al. Risk factors for medial meniscal pathology on knee MRI in older US adults: a multicentre prospective cohort study. Ann Rheum Dis 2011;70:1733-9. doi:10.1136/ard.2011.150052

12 Rytter S, Egund N, Jensen LK, Bonde JP. Occupational kneeling and radiographic tibiofemoral and patellofemoral osteoarthritis. J Occup Med Toxicol 2009;4:19. doi:10.1186/1745-6673-4-19.

13 Paxton ES, Stock MV, Brophy RH. Meniscal repair versus partial meniscectomy: a systematic review comparing reoperation rates and clinical outcomes. Arthroscopy 2011;27:1275-88. doi:10.1016/j. arthro.2011.03.088.

14 Meredith DS, Losina E, Mahomed NN, Wright J, Katz JN. Factors predicting functional and radiographic outcomes after arthroscopic partial meniscectomy: a review of the literature. Arthroscopy 2005;21:211-23. doi:10.1016/j.arthro.2004.10.003. 
15 Lysholm J, Gillquist J. Endoscopic meniscectomy. Int Orthop 1981;5:265-70. doi:10.1007/BF00271081.

16 Gillquist J, Oretorp N. Arthroscopic partial meniscectomy. Technique and long-term results. Clin Orthop Relat Res 1982;(167):29-33.

17 Hamberg P, Gillquist J. Knee function after arthroscopic meniscectomy. A prospective study. Acta Orthop Scand 1984:55:172-5. doi:10.3109/17453678408992331.

18 Kim JR, Kim BG, Kim JW, Lee JH, Kim JH. Traumatic and non-traumatic isolated horizontal meniscal tears of the knee in patients less than 40 years of age. Eur J Orthop Surg Traumatol 2013;23:589-93. doi:10.1007/s00590-012-1028-6.

19 Ghislain NA, Wei JN, LiYG. Study of the clinical outcome between traumatic and degenerative (non-traumatic) meniscal tears after arthroscopic surgery: a 4-years follow-up study. J Clin Diagn Res 2016;10:RC01-04.

20 Camanho GL, Hernandez AJ, Bitar AC, Demange MK, Camanho LF. Results of meniscectomy for treatment of isolated meniscal injuries: correlation between results and etiology of injury. Clinics (Sao Paulo) 2006;61:133-8. doi:10.1590/S1807-59322006000200008.

21 Vandenbroucke JP, von Elm E, Altman DG, et al. STROBE Initiative. Strengthening the Reporting of Observational Studies in Epidemiology (STROBE): explanation and elaboration. Epidemiology 2007;18:805-35. doi:10.1097/EDE.0b013e3181577511.

22 Thorlund JB, Christensen R, Nissen N, et al. Knee Arthroscopy Cohort Southern Denmark (KACS): protocol for a prospective cohort study. BMJ Open 2013;3:e003399. doi:10.1136/bmjopen-2013-003399.

23 Roos EM, Roos HP, Lohmander LS, Ekdahl C, Beynnon BD. Knee Injury and Osteoarthritis Outcome Score (KOOS)-development of a self-administered outcome measure. J Orthop Sports Phys Ther 1998;28:88-96. doi:10.2519/jospt.1998.28.2.88.

24 Collins NJ, Prinsen CA, Christensen R, Bartels EM, Terwee CB, Roos EM. Knee Injury and Osteoarthritis Outcome Score (KOOS): systematic review and meta-analysis of measurement properties. Osteoarthritis Cartilage 2016;24:1317-29. doi:10.1016/j.joca.2016.03.010

25 Roos EM, Roos HP, Lohmander LS. WOMAC Osteoarthritis Indexadditional dimensions for use in subjects with post-traumatic osteoarthritis of the knee. Western Ontario and MacMaster Universities. Osteoarthritis Cartilage 1999;7:216-21. doi:10.1053/joca.1998.0153.

26 Roos EM, Roos HP, Ekdahl C, Lohmander LS. Knee injury and Osteoarthritis Outcome Score (KOOS) - validation of a Swedish version. Scand I Med Sci Sports 1998;8:439-48. doi:10.1111/j.1600-0838.1998.tb00465.x.

27 Skou ST, Roos EM, Laursen MB, et al. A randomized, controlled trial of total knee replacement. N Engl J Med 2015;373:1597-606. doi:10.1056/NEJMoa1505467.

28 Frobell RB, Roos EM, Roos HP, Ranstam J, Lohmander LS. A randomized trial of treatment for acute anterior cruciate ligament tears. N Engl J Med 2010;363:331-42. doi:10.1056/NEJMoa0907797.

29 Kise NJ, Risberg MA, Stensrud S, Ranstam J, Engebretsen L, Roos EM. Exercise therapy versus arthroscopic partial meniscectomy for degenerative meniscal tear in middle aged patients: randomised controlled trial with two year follow-up. BM/ 2016:354:i3740. doi:10.1136/bmj.i3740.

30 Tubach F, Ravaud P, Baron G, et al. Evaluation of clinically relevant states in patient reported outcomes in knee and hip osteoarthritis: the patient acceptable symptom state. Ann Rheum Dis 2005;64:34-7. doi:10.1136/ard 2004.023028.

31 Ingelsrud LH, Granan LP, Terwee CB, Engebretsen L, Roos EM. Proportion of patients reporting acceptable symptoms or treatment failure and their associated KOOS values at 6 to 24 months after anterior cruciate ligament reconstruction: a study from the Norwegian Knee Ligament Registry. Am J Sports Med 2015;43:1902-7. doi:10.1177/0363546515584041
32 Anderson AF, Irrgang JJ, Dunn W, et al. Interobserver reliability of the International Society of Arthroscopy, Knee Surgery and Orthopaedic Sports Medicine (ISAKOS) classification of meniscal tears. Am / Sports Med 2011;39:926-32. doi:10.1177/0363546511400533.

33 Brittberg M, Winalski CS. Evaluation of cartilage injuries and repair. J Bone Joint Surg Am 2003;85-A(Suppl 2):58-69. doi:10.2106/00004623-200300002-00008.

34 Smith GD, Taylor J, Almqvist KF, et al. Arthroscopic assessment of cartilage repair: a validation study of 2 scoring systems. Arthroscopy 2005:21:1462-7. doi:10.1016/j.arthro.2005.09.007.

35 Paulsen A, Overgaard S, Lauritsen JM. Quality of data entry using single entry, double entry and automated forms processing--an example based on a study of patient-reported outcomes. PLoS One 2012;7:e35087. doi:10.1371/journal.pone.0035087.

36 Satterthwaite FE. An approximate distribution of estimates of variance components. Biometrics 1946;2:110-4. doi:10.2307/ 3002019.

37 Roos EM, Lohmander LS. The Knee injury and Osteoarthritis Outcome Score (KOOS): from joint injury to osteoarthritis. Health Qual Life Outcomes 2003;1:64. doi:10.1186/1477-7525-1-64

38 Haviv B, Bronak S, Kosashvili Y, Thein R. Arthroscopic meniscectomy of traumatic versus atraumatic tears in middle aged patients: is there a difference?Arch Orthop Trauma Surg 2016;136:1297-301. doi:10.1007/s00402-016-2504-y.

39 Roos H, Adalberth T, Dahlberg L, Lohmander LS. Osteoarthritis of the knee after injury to the anterior cruciate ligament or meniscus: the influence of time and age. Osteoarthritis Cartilage 1995;3:261-7. doi:10.1016/S1063-4584(05)80017-2

40 Thorlund JB, Hare KB, Lohmander LS. Large increase in arthroscopic meniscus surgery in the middle-aged and older population in Denmark from 2000 to 2011. Acta Orthop 2014:85:287-92. doi:10.3109/17453674.2014.919558.

41 Roos EM, Roos HP, Ryd L, Lohmander LS. Substantial disability 3 months after arthroscopic partial meniscectomy: A prospective study of patient-relevant outcomes. Arthroscopy 2000;16:619-26. doi:10.1053/jars.2000.4818.

42 Paradowski PT, Bergman S, Sundén-Lundius A, Lohmander LS, Roos EM. Knee complaints vary with age and gender in the adult population. Population-based reference data for the Knee injury and Osteoarthritis Outcome Score (KOOS). BMC Musculoskelet Disord 2006:7:38, doi:10.1186/1471-2474-7-38.

43 Sihvonen R, Paavola M, Malmivaara A, et al. Finnish Degenerative Meniscal Lesion Study (FIDELITY) Group. Arthroscopic partial meniscectomy versus sham surgery for a degenerative meniscal tear. N Engl J Med 2013;369:2515-24. doi:10.1056/NEJMoa1305189.

44 Sihvonen R, Englund M, Turkiewicz A, Järvinen TL. Finnish Degenerative Meniscal Lesion Study Group. Mechanical symptoms and arthroscopic partial meniscectomy in patients with degenerative meniscus tear: a secondary analysis of a randomized trial. Ann Intern Med 2016;164:449-55. doi:10.7326/M15-0899.

45 Roemer FW, Kwoh CK, Hannon MJ, et al Partial meniscectomy is associated with increased risk of incident radiographic osteoarthritis and worsening cartilage damage in the following year. Eur Radiol 2017:27:404-13. doi:10.1007/s00330-016-4361-z.

46 Brophy RH, Gray BL, Nunley RM, Barrack RL, Clohisy JC. Total knee arthroplasty after previous knee surgery: expected interval and the effect on patient age. J Bone Joint Surg Am 2014;96:801-5. doi:10.2106/JBJS.M.00105.

\section{Appendix: Supplementary material}

